\title{
A New Approach to Architecture of Sensor Networks for Mission-Oriented Applications
}

\author{
Chi Harold Liu ${ }^{\dagger}$, Kin K. Leung ${ }^{\dagger}$, Chatschik Bisdikian ${ }^{\ddagger}$, and Joel W. Branch ${ }^{\ddagger}$ \\ $\dagger$ Imperial College, London, SW7 2BT, U.K. \\ $\ddagger$ IBM T. J. Watson Research Center, Hawthorne, New York, 10532, U.S.A.
}

\begin{abstract}
In this paper, a novel mission-oriented sensor network architecture for military applications is proposed involving multiple sensing missions with varying quality of information (QoI) requirements. A new concept of mission QoI satisfaction index indicating the degree of satisfaction for any mission in the network is introduced. Furthermore, the $5 \mathrm{WH}$ (why, when, where, what, who, how) principle on the operational context of information is extended to capture the changes of QoI satisfaction indexes for mission admission and completion. These allow modeling the whole network as a "black box". With system inputs including the QoI requirements of the existing and newly arriving missions and output the QoI satisfaction index, the new concept of sensor network capacity is introduced and mathematically described. The QoI-centric sensor network capacity is a key element of the proposed architecture and aids controlling of admission of newly arriving missions in accordance with the QoI needs of all (existing and newly admitted missions). Finally, the proposed architecture and its key design parameters are illustrated through an example of a sensor network deployed for detecting the presence of a hazardous, chemical material.
\end{abstract}

Keywords: Wireless Sensor Networks, Mission-Oriented Applications, Sensor Network Capacity, Admission Control for Missions

\section{INTRODUCTION}

Continuing advances in sensors and sensor-supporting technologies including pervasive computing and communications capabilities reduce the cost of introducing and using smart autonomous wireless sensor networks (WSN) in the highly unpredictable and dynamic contexts of military operations [1]. Consequently, we are witnessing a considerable amount of research work regarding the deployment and operation of WSNs. To date, this research is mostly focusing on the "internal" operation of WSNs in areas such as energy-efficiency, coverage, routing topologies for efficient query and data dissemination, and so on [1]. In contrast, the areas considering the "external" relationships that WSNs have with the applications they support have seen significant less exposure. Such relationships pertain the information needs of the applications that sensors support and this paper specifically relates to the nascent area of quality of information (QoI) for wireless sensor networks.

Broadly speaking, QoI relates to the ability of available information to provide sufficient content at a reliable enough level to be fit-for-use by applications. It serves as a means to capture an application's information needs from the sensors. Information quality has been extensively studied in the context of enterprise information systems $[2,3]$, but has been studied sparsely in the area of wireless sensor networks [4]. For the purposes of this paper, we assume that QoI is characterized by number of attributes that allow assessment of the accuracy, timeliness, completeness, and so on, and the spatiotemporal relevancy of the information provided relatively to what is sought; QoI relevancy is the primary focus in this work.

The importance of QoI in military environments is, without a doubt, very high. The information collected from the sensor networks supports mission-critical decision making and action taking and the effectiveness of the latter depends on the QoI of the information used. Our research has been motivated by the synergistic

Further author information: (Send correspondence to Chi Harold Liu: E-mail: chiliu@imperial.ac.uk) Kin K. Leung: E-mail: kin.leung@imperial.ac.uk; Chatschik Bisdikian: E-mail: bisdik@us.ibm.com; Joel W. Branch: E-mail: branchj@us.ibm.com. 
benefits to be derived by "marrying" the objectives of the aforementioned traditional research about the internal operation of the WSNs with that of the external relationships that these networks have with the applications they support. Acknowledging the key role that QoI plays, this marriage gives rise to the novel research direction of QoI-aware management of WSNs. Specifically, in our research, we seek to develop the founding principles, including architectures and algorithmic mechanisms, that would enable managing the operation of WSNs to provide the varying levels of QoI expected by missions (the sensing applications) as these missions come and go dynamically.

To this end, this paper introduces a QoI-aware architecture and its key components, mathematically describes its main operational objectives, and highlights it through an exemplary use-case of a sensing system deployed for the detection of hazardous chemicals. The objective of the proposed QoI-aware architecture, which is the first such architecture to our knowledge, is to improve operational efficiencies of the entire system by optimally balancing between mission QoI, admission control for new missions, and sensor network capacities in the midst of the unpredictable nature of wireless communications and military operations. The absence of such balance would result in any combination of wasteful use of communication and computing resources, reduced admission rate for new missions, and increased rate of violations of the QoI of the missions accepted.

In [4], the 5WH (why, what, when, where, who, and how) principle was introduced as a means to consistently describe the information needs of an application and the information capabilities of sensor information providers. This principle gives us the opportunity to bridge between the QoI application requirements and the QoI-aware operation of sensor networks. Of particular interest in this paper are the when and what primitives which are used to capture the spatiotemporal properties of information (which is represented by the what primitive) of interest. For a given what (e.g., concentration of a specific chemical), that when (resp. where) relates to the time (resp. region) over which the what is sought by the application or the sensor information provider (the who primitive) can provide. The larger the overlap between the spatiotemporal properties between the information sought by applications and that provided by information providers the larger the degree of "relevancy" of the corresponding pieces of information becomes. For this reason, the when and where primitives are used to describe the spatiotemporal relevancy of information.

In support of our architecture, we introduce a new concept, QoI satisfaction index, which is used to indicate the degree of QoI satisfaction for any mission in the network, and further use this index to capture the change of QoIs for mission admission and completion. Later, we model the WSNs as a "black box", while considering as multiple QoI requirements as input variables and defined QoI satisfaction index as one single output. This model helps us mathematically analyze the so-called sensor network capacity which represents the maximum capability of the network to support the QoI requirements of applications. The sensor network capacity plays key role during the QoI-aware admission of newly arriving missions that seek service from the sensor network. When the QoI requirements of a new mission are such that its admission will violate the sensor network capacity, the mission may not be admitted, or, otherwise, its admission may result in degradation of the QoI level provided by the network to already existing missions. Summarizing, the contributions in this paper include:

(1) The introduction of the QoI satisfaction index.

(2) Extend the 5WH [4] principle and capture the change of QoIs for mission admission and completion.

(3) The introduction of the sensor network capacity and a proposal for general mathematical formulation for estimating it.

(4) A proposal for QoI-aware architecture for managing the admission of newly arriving missions utilizing the aforementioned QoI satisfaction index, abrupt changes of QoIs for mission arrival and completion, and sensor network capacity.

The rest of the paper is organized as follows. After summarizing the related works in Section 2, the new concept of QoI satisfaction index and change of QoIs for mission admission and completion are presented in Section 3. A thorough description of system modeling that includes the black box representation, sensor network capacity estimation, and admission control for missions are reported in Section 4. Last, a detailed chemical detection example to illustrate the whole idea is in Section 5. Finally, conclusions are drawn in Section 6. 


\section{RELATED WORK}

Despite of endeavors for defining QoI [2, 3], until recently, work in [4] proposed a conceptual framework to facilitate the dynamic binding of sensor information producers and consumers in QoI-aware manner. The framework represents information needs and capabilities according to the $5 \mathrm{WH}$ principle to facilitate information producers to categorize the quality attributes of their information in application-agnostic manner while permit information consumers to calculate QoI in application-specific way. From sensor architecture perspective, not much research has been done to study the interactions among mission applications and network resources. Recent work in [5] proposed a simple model to characterize the information loss due to network delays and buffer overflows in order to make admission control decisions for new applications. Work in [6] presented a framework that incorporates multiple factors (e.g., resource contention, the state of phenomena, etc.) into the QoI to assure the delivered quality. By modeling the probability of delivery time from source to sink nodes and the probability of a data unit to be lost due to buffer constraints under CSMA/CA MAC protocol, this paper proposed a mathematical framework calculating the resource availability distribution for new applications.

Meanwhile, researchers have been focusing on WSNs middleware design [7] that use a notion of quality of obtained information and do sensor management with the aim to satisfy certain information quality bounds. MiLAN [8] addresses the question of how to guarantee in the presence of resource constraints; however, it does not have a clear and quantitative QoI definition. In QUASAR [9], data quality is defined as application error tolerance and approximated as the cases when data is lost or unavailable due to resource limitations. The Midfusion [10] architecture separates information fusion into two levels, sensor and application, each of which is represented by a Bayesian network [11]. However, in making the decision of which set of sensors to choose, the Midfusion architecture only considers the available applications, their structure, and available sensors, but not the dynamic status of WSNs. Furthermore, the sensor allocation is changed only when the set of applications, their requirements, or the set of available sensors are changed. In [12], they proposed a framework which made dynamic resource allocations according to the current observed state of the environment for the quality of service (QoS) requirements of the applications. The goal of the framework was to choose an optimal set of sensors to monitor a system while maintaining a certain level of the QoS obtained. The issue of changing network state was studied in [13] where information quality is satisfied by choosing the most appropriate sensor nodes and sensor modalities for the current state of the network. Recent works in $[14,15]$ are more related to this paper in that they tackle the connection admission control problem in wireless mesh networks to estimate the route capacity associated with each source/gateway pair.

\section{QOI SATISFACTION INDEX}

Consider a WSN that comprises of $N$ sensor nodes, denoted by $S=\left\{s_{i} \mid i=1,2, \ldots, N\right\}$ and let the subset of sensors $\mathbf{s}_{q} \in S$ be used by mission $q$ as data sources (not necessarily exclusively though). Mission $q$ requires the monitoring of a specific feature such as temperature, event location, density of a hazardous chemical, and so on. Each feature is associated with multiple QoI requirements, namely, accuracy $\tau_{q}^{r}$ (which consists of error bound requirement $\epsilon_{q}^{r}$ and confidence requirement $\delta_{q}^{r}$ ), completeness $c_{q}^{r}$, and timeliness $d_{q}^{r}$ (such as mission response time). The sensors in $\mathbf{s}_{q}$ monitor for these features by taking measurements $x_{q}^{i}(t), \forall i \in \mathbf{s}_{q}$ periodically for each feature at either discrete or continuous time instances. Furthermore, a data fusion function $\phi_{t}[\cdot]$ is used at each sensor node to process these measurements, so that one single measurement output per-data source $\kappa^{i}=\phi_{t}\left[x_{q}^{i}(t)\right]$ is generated. A number of destinations/sinks are responsible for collecting these measurements from multiple data sources, so that the data will travel through the multi-hop WSNs back to one/multiple sinks. At the sink side, we assume another information fusion function $\zeta_{i}\{\cdot\}$ is used to merge these measurements $\kappa^{i}, \forall i \in \mathbf{s}_{q}$ and generate a final sensor reading as:

$$
\theta_{q}^{a}=\zeta_{i}\left\{\kappa^{i}\right\}=\zeta_{i}\left\{\phi_{t}\left[x_{q}^{i}(t)\right]\right\}, \forall i \in \mathbf{s}_{q} \text { and } \forall t .
$$

where the superscript $a$ denotes the actual measured value. Therefore, the mission generator is aware of this measured value $\theta_{q}^{a}$ from multiple sources, where in our QoI definition $\theta$ represents accuracy $\tau$, completeness $c$, and timeliness $d$. Finally, we assume the "correct" mission information is $z_{q}$, for instance, the "correct" value for the feature of interest, e.g., the density of a chemical. 


\subsection{QoI Satisfaction Index}

Given the QoI requirement of mission $q$, i.e., accuracy (error bound $\epsilon_{q}^{r}$ and confidence $\delta_{q}^{r}$ ), completeness $c_{q}^{r}$, and timeliness $d_{q}^{r}$, and measured values $\epsilon_{q}^{a}, \delta_{q}^{a}, c_{q}^{a}, d_{q}^{a}$ desired to obtained at sinks, we want to identify the degree of QoI satisfaction the WSN can support for a particular mission $q$. Therefore, we define a set of QoI satisfaction indexes, comprised of each QoI requirement, $\mathbf{I}_{q}^{\tau}, \mathbf{I}_{q}^{c}$, and $\mathbf{I}_{q}^{d}$, as the ratio between actual measured value and requirement, or vice versa. In the following context, we first use accuracy as an example to demonstrate how to obtain the QoI satisfaction index, and then we show how to merge multiple QoI satisfaction indexes into one single output.

If $z_{q}$ denotes the "correct" value of information, the satisfactory QoI condition for accuracy is given by:

$$
\tau_{q}^{a}=\operatorname{Pr}\left(\left|z_{q}-\zeta_{i}\left\{\phi_{t}\left[x_{q}^{i}(t)\right]\right\}\right| \leq \epsilon_{q}^{r}\right) \geq \delta_{q}^{r}, \forall i \in \mathbf{s}_{q} \text { and } \forall t .
$$

In this way, we explicitly incorporate the accuracy requirement of a mission into actual sensor measurements in a probabilistic manner.

We define the QoI satisfaction index for accuracy $\mathbf{I}_{q}^{\tau}$ as the ratio between its desired value of the QoI level $\tau_{q}^{r}$ and the one attained $\tau_{q}^{a}$ due to limited sensing and communications abilities:

$$
\mathbf{I}_{q}^{\tau}=\frac{\tau_{q}^{r}}{\tau_{q}^{a}}=\frac{\delta_{q}^{r}}{\operatorname{Pr}\left(\left|z_{q}-\zeta_{i}\left\{\phi_{t}\left[x_{q}^{i}(t)\right]\right\}\right| \leq \epsilon_{q}^{r}\right)} \in(0,+\infty) .
$$

Note that when the QoI requirement (accuracy in this case) of mission $q$ is satisfied if and only if $\mathbf{I}_{q}^{\tau} \in(0,1]$. This QoI satisfaction index not only represents the sensing quality at a selected a group of sensors $\mathbf{s}_{q}$, but also reflects the communications quality of multi-hop WSNs for the reporting route, when it is measured at the sink side. This is important because QoI relies on two parts: information sensing on multiple data sources, and information delivery/reporting through multi-hop WSNs that may incur further packet loss, delay or damage.

We similarly define the other two QoI satisfaction indexes for completeness and timeliness:

$$
\mathbf{I}_{q}^{c}=\frac{c_{q}^{r}}{c_{q}^{a}} \in(0,1], \quad \mathbf{I}_{q}^{d}=\frac{d_{q}^{a}}{d_{q}^{r}} \in(0,1]
$$

where the range of the indexes are given for when the QoI requirements of mission $q$ are satisfied. Later on, a single QoI satisfaction index that incorporates all three QoI requirements is introduced. As an illustrative example, one may simply use the maximum of three ratios as:

$$
\mathbf{I}_{q}=\max \left(\mathbf{I}_{q}^{\tau}, \mathbf{I}_{q}^{c}, \mathbf{I}_{q}^{d}\right)
$$

For any mission $q, \mathbf{I}_{q} \in(0,1]$ means none of the QoI requirements are violated and it serves as a bottom line for any satisfactory task.

\subsection{Abrupt Changes of QoIs for Mission Arrival and Completion}

We notice that the operational context of missions' multiple QoI requirements may require overlapping geographical and time information, and they use different amounts of resources to execute the tasks. Research on 5WH-based QoI concept [4] provides this multi-dimensional information, specifically as (why, when, where, what, who, how), to capture the information needs in terms of space, time and network resources. However, the partial resource contention between new mission and existing missions may cause abrupt changes on defined QoI satisfaction index. This is because a new mission admission will cause abrupt degradation of the QoI satisfaction index for existing missions due to sharing nature of network resources, such as power, sensing device, bandwidth, etc. Furthermore, when existing a mission completes, the released resources will be utilized by ongoing missions so that ongoing missions' QoI satisfaction indexes will experience abrupt improvements. Clearly, to effectively manage the admission of missions, we need to predict sufficiently well the impact that the arrivals and completion of missions have to the QoI satisfaction indexes. 


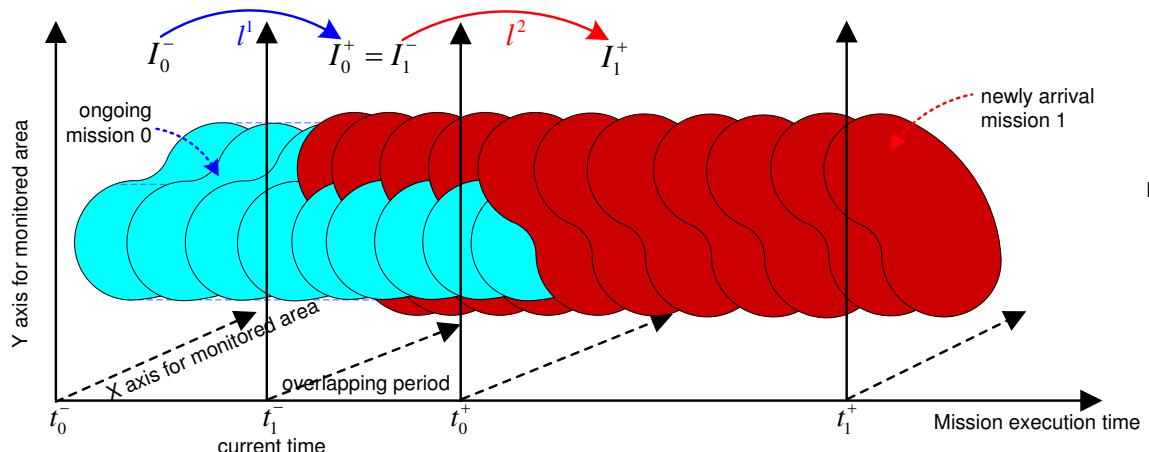

(a)

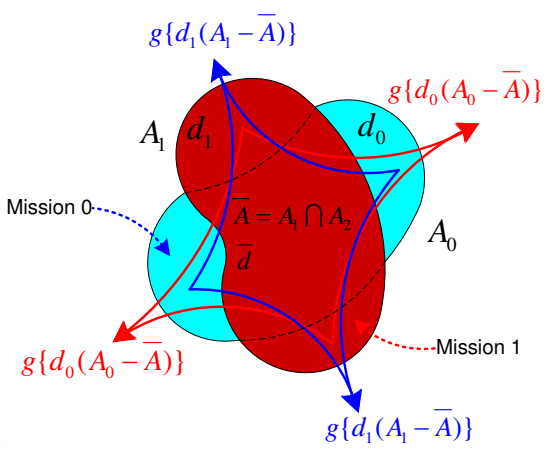

(b)

Figure 1. An example to illustrate the abrupt QoI satisfaction index changes due to mission arrival and completion. (a) mission 0 and mission 1 require QoI in overlapping areas and time periods (b) an example to show how network resources are shared by parallel missions, since sensors may serve as relays to forward the data under the broadcasting assumption of WSNs.

To illustrate our ideas, we consider the following example in Figure 1, where mission 1 is arriving at the WSN that already services mission 0; for simplicity, a 2-D example is considered. The operational context of QoI requirements for mission 0 and mission 1 partially content with each other in overlapping area $A_{0}$ and $A_{1}$, and mission execution time $\left[t_{0}^{-}, t_{0}^{+}\right]$and $\left[t_{1}^{-}, t_{1}^{+}\right]$respectively, as in Figure 1(a) and Figure 1(b). Further assume $d_{0}, d_{1}, \bar{d}$ denote the sensor densities for area $A_{0}, A_{1}$ and $\bar{A}=A_{0} \cap A_{1}$ respectively. As shown in Figure 1(a), during $\left[t_{1}^{-}, t_{0}^{+}\right]$, if mission 1 is admitted, some sensors would execute two tasks simultaneously, and thus network resources would be shared and the degree of QoI satisfaction would degrade. However, during $\left[t_{0}^{+}, t_{1}^{+}\right]$, due to mission 0's completion, QoI satisfaction for mission 1 would improve. Therefore, we can see two abrupt QoI satisfaction index changes. Let $l^{1}$ and $l^{2}$ denote the two ratios of QoI index at the change points $t_{1}^{-}$and $t_{0}^{+}$ respectively. We write $\mathbf{I}_{0}^{-}$(resp. $\mathbf{I}_{0}^{+}$) for the QoI satisfaction index for mission 0 before (resp. after) admitting mission 1 into the network. Likewise, we write $\mathbf{I}_{1}^{-}$(resp. $\mathbf{I}_{1}^{+}$) for the QoI satisfaction index experienced by mission 1 before (resp. after) mission 0 terminates and releases its resources. Note that $\mathbf{I}_{0}^{+}=\mathbf{I}_{1}^{-}$holds because the sink nodes will integrate multiple concurrent missions' QoI satisfaction indexes into one single value (average for instance), i.e., at a particular time, there is only one satisfaction index for all concurrent missions. Therefore, our ratios to capture the index changes could be defined as:

$$
l^{1}=\frac{\mathbf{I}_{0}^{+}}{\mathbf{I}_{0}^{-}}, \quad l^{2}=\frac{\mathbf{I}_{1}^{+}}{\mathbf{I}_{1}^{-}} .
$$

The overall index change could be expressed as another ratio between QoI index after admitting mission 1 and QoI index before admitting mission 1, as:

$$
\text { QoI Index Change } l=\frac{\text { weight }\left\{\mathbf{I}_{1}^{+}, \mathbf{I}_{1}^{-}\right\}}{\operatorname{weight}\left\{\mathbf{I}_{0}^{+}, \mathbf{I}_{0}^{-}\right\}}=\frac{\alpha_{1} \mathbf{I}_{1}^{+}+\left(1-\alpha_{1}\right) \mathbf{I}_{1}^{-}}{\alpha_{0} \mathbf{I}_{0}^{+}+\left(1-\alpha_{0}\right) \mathbf{I}_{0}^{-}}=\frac{\alpha_{1} \frac{\mathbf{I}_{1}^{+}}{\mathbf{I}_{1}^{-}}+\left(1-\alpha_{1}\right)}{\alpha_{0} \frac{\mathbf{I}_{0}^{+}}{\mathbf{I}_{1}^{-}}+\left(1-\alpha_{0}\right) \frac{\mathbf{I}_{0}^{-}}{\mathbf{I}_{1}^{-}}},
$$

where weight factors $\alpha_{0}$ and $\alpha_{1}$ are introduced here to balance time importance at change point. Using the fact that $\mathbf{I}_{0}^{+}=\mathbf{I}_{1}^{-}$, (7) becomes:

$$
\text { QoI Index Change } l=\frac{\alpha_{1} \frac{\mathbf{I}_{1}^{+}}{\mathbf{I}_{1}^{-}}+\left(1-\alpha_{1}\right)}{\alpha_{0}+\left(1-\alpha_{0}\right) \frac{\mathbf{I}_{0}^{-}}{\mathbf{I}_{0}^{+}}}=\frac{\alpha_{1} l^{2}+\left(1-\alpha_{1}\right)}{\alpha_{0}+\left(1-\alpha_{0}\right) \frac{1}{l^{1}}} .
$$

In the following discussions, we derive $l^{1}$ and $l^{2}$. As mentioned, for time period $\left[t_{1}^{-}, t_{0}^{+}\right]$, mission 0 could not sustain the original QoI satisfaction level, since network resources (sensors, power, time, etc.) are shared with 
mission 1 in both overlapping area $\bar{A}$ and the rest of non-overlapping areas. This is because if we assume a broadcasting nature of WSNs, sensors deployed in non-overlapping area $A_{1}-\bar{A}$ will forward their data through sensors in area $A_{0}-\bar{A}$, as seen in Figure 1(b). Therefore, communications resources at $A_{0}-\bar{A}$ will no longer be used by mission 0 only, so that part of original allocated resources will be used for the purpose of mission 1's data forwarding. Similar judgement applies to overlapping area $\bar{A}$.

To capture the resource-sharing nature of WSNs, we introduce a non-decreasing utility function $g\{\cdot\}$. This utility function maps the network resources in a certain area to the degree of QoI satisfaction change, i.e., if more resources are shared by multiple concurrent missions, higher degree of QoI satisfaction loss is expected. The input parameter of this function could be the number of sensors, sensor locations, remaining power, bandwidth, etc. It can be obtained through specific network protocol modeling or from empirical measurements; this is beyond the scope of the current paper. For illustrative purposes, let us use the number of sensors to demonstrate the derivation of index change. The number of sensor that carry information for mission 1 through the area $A_{0}-\bar{A}$ is $d_{0}\left(A_{0}-\bar{A}\right)$ and the number of sensors that are shared by both missions is $\bar{d} \bar{A}$. Thus, $g\left\{d_{0}\left(A_{0}-\bar{A}\right)\right\}$ and $g\{\bar{d} \bar{A}\}$ can be used to represent the percentages of QoI satisfaction loss for these two parts respectively. Therefore, the expected QoI satisfaction index for mission 0 if mission 1 is to be admitted is given by:

$$
\mathbf{I}_{0}^{+}=\beta_{1} \cdot g\{\bar{d} \bar{A}\} \cdot \mathbf{I}_{0}^{-}+\left(1-\beta_{1}\right) \cdot g\left\{d_{0}\left(A_{0}-\bar{A}\right)\right\} \cdot \mathbf{I}_{0}^{-},
$$

where $\beta_{1}$ is the weight factor to indicate geographical importance or priority. From (9), we derive $l^{1}$ as:

$$
l^{1}=\frac{\mathbf{I}_{0}^{+}}{\mathbf{I}_{0}^{-}}=\beta_{1} \cdot g\{\overline{d A}\}+\left(1-\beta_{1}\right) \cdot g\left\{d_{0}\left(A_{0}-\bar{A}\right)\right\} .
$$

Now, we proceed to derive the ratio $l^{2}$ for QoI change when mission 0 completes leaving mission 1 with full resources. Both overlapping and non-overlapping areas will cause this index change. In $\bar{A}$, mission 1 will re-use the network resources previously occupied by mission 0 , so it is expected that over this area the QoI satisfaction index will change back to $\mathbf{I}_{0}^{-}$, i.e., the QoI satisfaction index that mission 0 previously experienced prior to the admission of mission 1. Meanwhile, for area $A_{1}-\bar{A}$, QoI satisfaction index will improve from $\mathbf{I}_{1}^{-}$to $\frac{1}{g\left\{d_{1}\left(A_{1}-\bar{A}\right)\right\}} \mathbf{I}_{1}^{-}$, since mission 0 will no longer use sensor resources in this area to forward its own data. We have:

$$
\mathbf{I}_{1}^{+}=\left(1-\beta_{2}\right) \cdot \mathbf{I}_{0}^{-}+\beta_{2} \cdot \frac{1}{g\left\{d_{1}\left(A_{1}-\bar{A}\right)\right\}} \cdot \mathbf{I}_{1}^{-},
$$

or,

$$
l^{2}=\frac{\mathbf{I}_{1}^{+}}{\mathbf{I}_{1}^{-}}=\left(1-\beta_{2}\right) \cdot \frac{\mathbf{I}_{0}^{-}}{\mathbf{I}_{1}^{-}}+\beta_{2} \cdot \frac{1}{g\left\{d_{1}\left(A_{1}-\bar{A}\right)\right\}}=\left(1-\beta_{2}\right) \cdot \frac{1}{l^{1}}+\beta_{2} \cdot \frac{1}{g\left\{d_{1}\left(A_{1}-\bar{A}\right)\right\}},
$$

where $\beta_{2}$ is the weight factor to indicate geographical importance or priority and we replace back $\mathbf{I}_{1}^{-}=\mathbf{I}_{0}^{+}$in the expression.

\section{SYSTEM MODELING}

\subsection{The Black Box Representation}

As shown in Figure 2, missions are assigned through some sensor nodes to the WSN with multiple QoI requirements. Sensor measurements from multiple data sources are reported back to the sinks through multi-hop WSNs. Therefore, at the sink, the QoI satisfaction index could be observed for any given mission. This index is achieved by protocol layers 1, 2, and 3 of each node, which ensure the accuracy and timeliness of packet delivery (while also reducing packet loss) of sensed information to maintain QoI requirements. However, the multi-hop operational characteristics and detailed protocol models used along the entire route of communication are not always transparent to the sink's upper layers, and protocol layers always have different operation time scales. Furthermore, in a WSN, there is not always a central authority controlling the network. Therefore, to overcome 


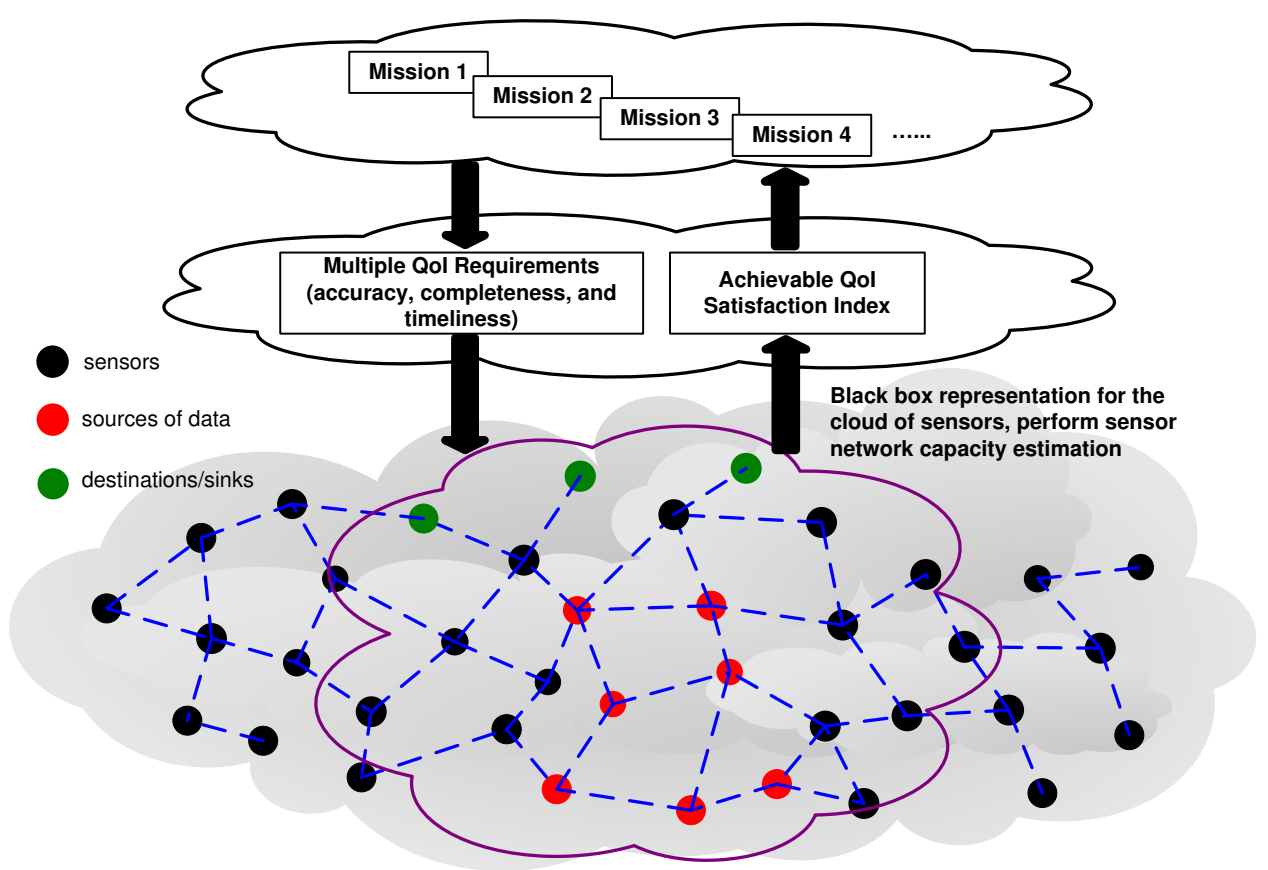

Figure 2. The overall architecture of the proposed mission-oriented WSN where it is treated as a "black box", where inside the black box, there are source sensors, relays in the multi-hop wireless communications, and sinks, which are involved in either data collection or data reporting for sensor measurements. When missions express their QoI requirements into the WSN, in response, it performs sensor network capacity estimation to inform the mission the degree of QoI satisfaction.

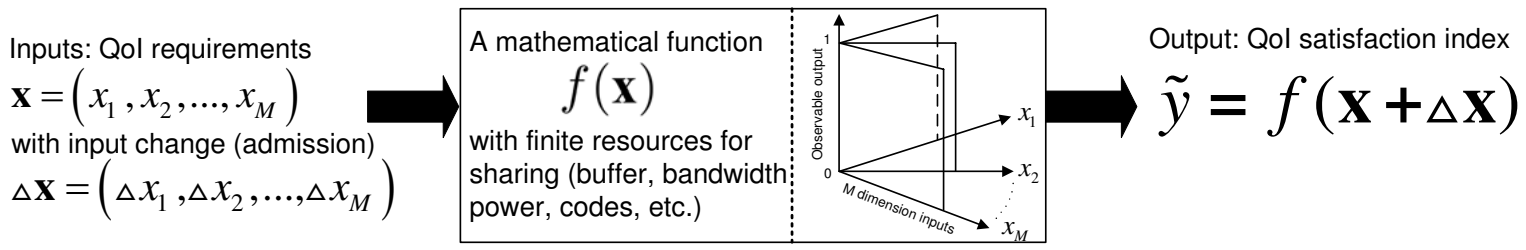

Figure 3. The mathematical representation of the black box, which are resource manager (buffer, bandwidth, power, codes, etc.) and sensing abilities. Mission's $M$ dimension QoI requirements serve as input variables, and observable QoI satisfaction index is a single output.

these difficulties, we model the WSN as a "wireless system," or a "black box," for the cloud of sensors, i.e., inside the black box, there are sources of data, relays, and sinks, which are involved in collecting and reporting sensor measurements. Finite resources are shared by multiple missions within the black box as shown in Figure 3 . These resources include, but are not limited to, devices, time, buffers, bandwidth, power, etc. The system inputs are missions' QoI requirements, and by monitoring the resource occupancy as an output, or QoI satisfaction index, we want to identify the potentially limiting resources and estimate the sensor network capacity. Here, we formally define sensor network capacity as follows:

DEFINITION 1. Sensor network capacity corresponds to the overall maximum QoI satisfaction index a WSN can support for any combination of missions with different QoI requirements. It can be interpreted as a time-varying marginal capability beyond which all running missions' QoI can no longer be maintained. This capacity can be analyzed in terms of maximum information accuracy, completeness, smallest information gathering delay, and/or the maximum number of concurrent missions the network can support at any given time. 


\subsection{Mathematical Representation of WSNs}

Without loss of generality, we use a mathematical function $f$ to represent the "black box" to map inputs to an output. It is worth noting that $f$ is usually a complicated function, which generally does not have close-form expressions. Let vector $\mathbf{x}=\left(x_{1}, x_{2}, \ldots, x_{M}\right)$ denote $M$ input variables, and $y=f(\mathbf{x})$ denote the output degree of resource occupancy. The ratio of QoI index at the change point due to mission admission or completion captures the abrupt QoI satisfaction index change. These consist of $M+1$ dimensional space as shown in Figure 3, where $M$ input dimensions may not be orthogonal but should represent the required mission's QoI requirements. To overcome the difficulties of close-form representation of $f$, we use Taylor expansion [16] to approximate this resource manager. We further characterize the potential new mission admission as an input change $\Delta \mathbf{x}=\left(\Delta x_{1}, \Delta x_{2}, \ldots, \Delta x_{M}\right)$ into the resource manager, which will result in an output change of $\tilde{y}=f(\mathbf{x}+\Delta \mathbf{x})$. Then, if we only take the first and second order partial derivatives of $f$, we have the following approximation,

$$
\tilde{y}(\mathbf{x}+\Delta \mathbf{x}) \approx f(\mathbf{x})+\sum_{i=1}^{M} f_{x_{i}}^{\prime} \Delta x_{i}+\frac{1}{2 !}\left\{\sum_{i=1}^{M} f_{x_{i}}^{\prime \prime} \Delta x_{i}^{2}+\sum_{i=1}^{M} \sum_{j \neq i} f_{x_{j} x_{i}}^{\prime} \Delta x_{i} \Delta x_{j}\right\},
$$

where we denote $f_{x_{i}}^{\prime}=\frac{\partial f}{\partial x_{i}}, f_{x_{i}}^{\prime \prime}=\frac{\partial f^{2}}{\partial x_{i}^{2}}, f_{x_{j} x_{i}}^{\prime \prime}=\frac{\partial f^{2}}{\partial x_{j} \partial x_{i}}$. Before we proceed, we need to prove the validity of neglecting all higher order Taylor series. This is because the sensor network capacity is usually much larger than the resource demands of one single mission. And thus, the admission or completion of one mission does not drastically change the overall mission performances. Furthermore, any change in the overall mission performances (due to the admission or completion of one mission) is adequately reflected in the measured output before the next admission event.

The construction of $M+1$ dimensional space is achieved and maintained by monitoring enough missions so that whenever there is an admission or completion event, the current network status ( $M$ input variables) updates and also the corresponding output QoI satisfaction index. Through updating this space periodically, we know the shape of the curve produced by $f(x)$. Interestingly, if we further examine only one dimension of input variable and the corresponding output, we would expect trends such as that in Figure 4(a) and Figure 4(c).

As the QoI definition is introduced as accuracy, completeness, and timeliness in this paper, input variables could be those requirements accordingly $\left(\tau_{q}^{r}, c_{q}^{r}, d_{q}^{r}\right)$. The curve should be non-decreasing, i.e., given more stringent QoI requirement as the input variable, higher QoI satisfaction index (in other words, lower satisfaction level) should be expected. Therefore, the curve reaches an upper bound when QoI satisfaction index $\mathbf{I}_{q}=1$. Interestingly, when the monitored output statistics reach 1, the WSN reaches it capacity, and this is exactly what we have introduced as sensor network capacity, which closely relates to QoI requirements. We are interested in analyzing this capacity mathematically. Moreover, due to the stochastic nature of WSNs and dynamic QoI requirements for different missions, the shape of the curve will change over time, and even given fixed QoI inputs, the curve will change because resource allocation schemes at each sensor node will adapt with radio (and other resource-related) conditions. Nevertheless, our framework, or the black box characterization, and Taylor expansion, is always completely transparent to lower layers, and thus as long as sufficient statistics collection is performed, we can safely construct this $M+1$ dimensional space and accurately estimate sensor network capacity.

\subsection{Admission Control for Missions}

As shown in Figure 2, the proposed mission-oriented WSN architecture is a request-and-response process, where missions push QoI requests into the WSN, and based on the previous QoI satisfaction indexes observed and sensor network capacity predicted, the WSN informs the mission of the degree of QoI satisfaction the network can provide. If QoI for the new and existing missions cannot be satisfied, the new mission is rejected. This architecture is important because the mission can be made aware of the QoI it may receive before jeopardizing its own success, and potentially that of other missions, before entering the network.

Admission control for missions is the core function of the proposed QoI-aware architecture that uses predicted sensor network capacity $\mathbf{c}_{t}^{\max }$, which can be a scalar or a $P$-dimensional vector. When it is a vector, it could be the 
maximum supportable QoI requirements, namely, maximum accuracy confidence $\delta_{t}^{\max }$, maximum completeness $c_{t}^{\max }$, and minimum delay $d_{t}^{\max }$, or simply the maximum number of missions $n_{t}^{\max }$ the network can support. It is worth noting that this capacity can be obtained through numerical analysis after the Taylor expansion on each dimension of the $M+1$ dimensional space. The procedure is to explicitly set the $\mathrm{y}$-axis equal to 1 , or the maximum supportable QoI satisfaction index $\mathbf{I}_{t}$ equal to 1 , and we obtain the corresponding $\mathrm{x}$-axis value, the capacity, as:

$$
\mathbf{c}_{t}^{\max }=\left\{n_{t}^{\max }, \delta_{t}^{\max }, c_{t}^{\max }, d_{t}^{\max }, \ldots\right\} \in \Re^{P} .
$$

Now we come to the admission decision-making phase when a new mission $q$ requests to monitor the same feature(s) but with different QoI requirements. The proposed algorithm runs at the sink before assigning the task down to any sensor node(s). The first step is to use the earlier derived ratio $l$ in (8) to capture the abrupt index change due to this new mission's arrival and later on, the completion of existing missions. Specifically, suppose the current average QoI satisfaction index for all missions is $\mathbf{I}_{0}$, then the impacts of admitting one more mission on existing one's QoI could be captured by $l \cdot \mathbf{I}_{0}$, or the degraded QoI satisfaction level. Then, the admission decision variable $\Upsilon_{q}$ is chosen according to the following conditions,

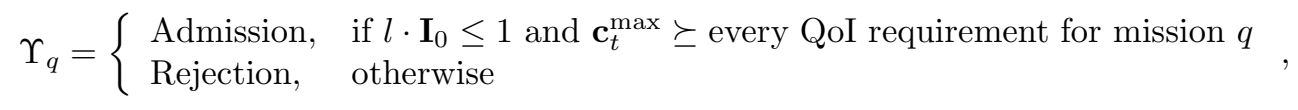

where notation $\succeq$ denotes the element-by-element comparison.

\section{A CHEMICAL DETECTION EXAMPLE}

In this section, we use a chemical detection example to illustrate the usefulness of our framework. Chemical detection tasks usually require accurate monitoring (in regards to QoI), i.e., accurate detection probability $\delta_{q}^{r}$ with error bound $\epsilon_{q}^{r}$. For simplicity, we neglect all other QoI requirements in this example.

\subsection{QoI Parameter}

As defined in (3), the QoI satisfaction index for accuracy could be introduced in a probabilistic manner, as the ratio between required confidence bound and actual measurement confidence. In this case, since all other QoI parameters are neglected, we have the final QoI satisfaction index for mission $q$ as:

$$
\mathbf{I}_{q}=\mathbf{I}_{q}^{\tau}=\frac{\delta_{q}^{r}}{\operatorname{Pr}\left(\left|z_{q}-\zeta_{i}\left\{\phi_{t}\left[x_{q}^{i}(t)\right]\right\}\right| \leq \epsilon_{q}^{r}\right)} \in(0,+\infty) .
$$

For satisfactory QoI, we require $\mathbf{I}_{q}=\mathbf{I}_{q}^{r} \leq 1$.

\subsection{System Modeling}

In this section, we use $M=2$ as an example to illustrate the concept of the black box characterization as well as how to use Taylor expansion to estimate the sensor network capacity. The inputs are chosen as the number of ongoing missions $n(t)$, and the worst case confidence bound $\delta_{w}^{r}(t)$. By "worst case", we mean the maximum confidence bound requirement among all ongoing chemical detection tasks in the network, i.e.,

$$
\delta_{w}^{r}(t)=\max _{\forall q} \delta_{q}^{r}
$$

This dimension of input gives the highest QoI requirement the network should have to support. In other words, as long as the worst case confidence bound is satisfied, all other missions' less stringent QoI requirements will be satisfied. This is due to the shared nature of wireless networks, or in our black box, one single statistic represents all current missions' QoI status. Hence, the network status is wholly represented by $\left(n(t), \delta_{w}^{r}(t)\right)$. Furthermore, in our black box representation, we use observable worst case QoI satisfaction index $\mathbf{I}_{w}(t)$ as an output. Again, 


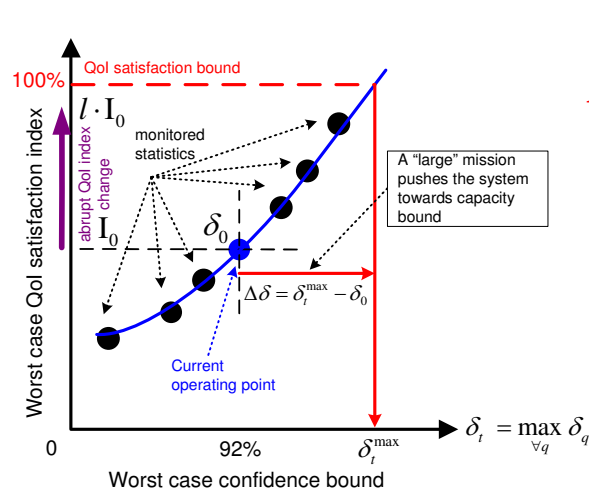

(a)

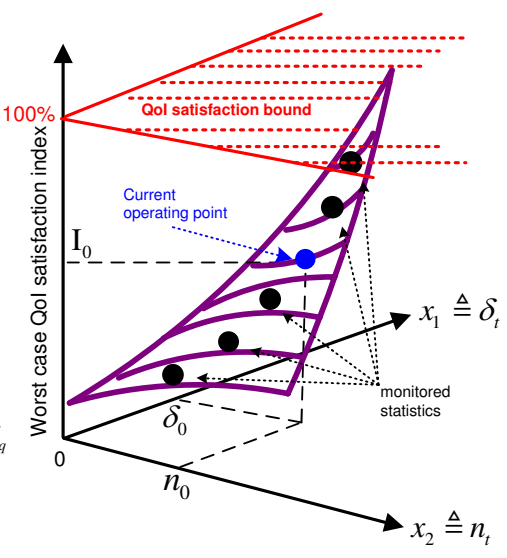

(b)

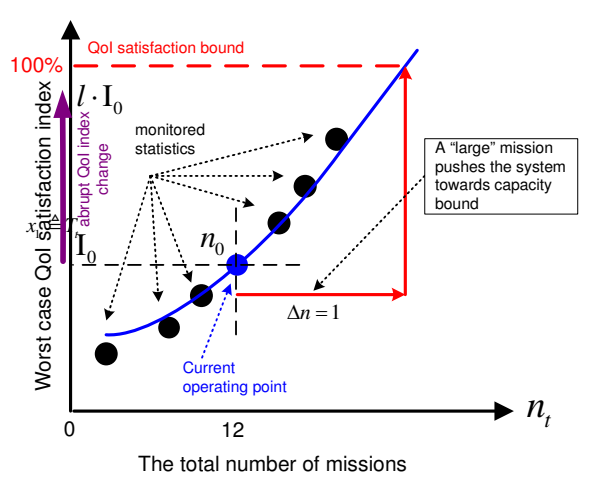

(c)

Figure 4. (a) An example of how to obtain sensor network capacity in terms of maximum supportable confidence bound $\delta_{t}^{\max }$ on dimension $x_{1}$ for accuracy. (b) Three dimension space representation of the black box, (c) An example of how to obtain sensor network capacity on dimension $x_{2}$.

by "worst case", we mean the maximum QoI satisfaction index among all running chemical detection tasks, which in turn, represents the degree of worst case supported QoI level:

$$
\mathbf{I}_{w}(t)=\max _{\forall q} \mathbf{I}_{q}
$$

For simplicity, we use $\left(n(t), \delta_{w}^{r}(t)\right), \mathbf{I}_{w}(t)$ and $\left(n_{t}, \delta_{t}\right), \mathbf{I}_{t}$ interchangeably. Therefore, the black box is characterized by:

$$
\mathbf{I}_{t}=f\left(n_{t}, \delta_{t}\right),
$$

where $\mathbf{I}_{t}, n_{t}$, and $\delta_{t}$ constitute a three-dimensional observation space. In this space, input variables are consistently updated due to mission arrivals or completions in real-time, and the corresponding output worst case QoI satisfaction index changes accordingly. The importance of using such a representation is two-fold. First, it is completely transparent not only to lower protocol layers but also all operation and control algorithm details. Second, and more importantly, the impact of newly admitted chemical detection tasks are sufficiently reflected as the output worst case QoI satisfaction index. This is because the network and sensing resources are shared with all missions, and thus any new mission will consume some resources of existing ones, which in turn, is reflected as the QoI satisfaction index change. However, as long as the worst case QoI satisfaction index falls between $(0,1]$, all missions will gain satisfactory QoI levels.

\subsection{Estimation of Sensor Network Capacity}

Following the WSN modeling as a black box where inputs are accuracy requirement and number of missions, and output reflects the degree of QoI satisfaction, in this section, we mathematically identify the sensor network capacity in terms of: the maximum confidence bound, or, $\mathbf{c}_{t}^{\max }=\delta_{t}^{\max }$.

We assume that the WSN operates at status $\left(n_{0}, \delta_{0}\right)$ at time $t_{0}$ when the new mission arrives for an admission decision, i.e., there are $n_{0}$ existing missions with worst case guaranteed confidence $\delta_{0}$, as shown in Figure 4(b). Then our black box is represented by,

$$
\mathbf{I}_{0}=f\left(n_{0}, \delta_{0}\right) .
$$

In order to estimate the sensor network capacity, we thereby assume that a "large" chemical detection task requires admission into the network, where "large" means a very stringent QoI requirement that pushes our system to the capacity bound: $\mathbf{I}_{+} \Rightarrow 1$. As shown in Figure 4(a) and Figure 4(c), this large new mission arrival corresponds to an input change $\Delta \delta=\delta_{t}^{\max }-\delta_{0}$ and $\Delta n=1$, and for the expected output change,

$$
\mathbf{I}_{+}\left(n_{t}^{\max }, \delta_{t}^{\max }\right)=1
$$


should have to hold. Therefore, we rewrite Taylor expansion (13) as,

$$
\mathbf{I}_{+}\left(n_{t}^{\max }, \delta_{t}^{\max }\right)=\mathbf{I}_{0}+\left.\Delta n f_{n_{t}}^{\prime}\right|_{t=t_{0}}+\left.\Delta \delta f_{\delta_{t}}^{\prime}\right|_{t=t_{0}}+\left.\frac{\Delta n^{2}}{2} f_{n_{t}}^{\prime \prime}\right|_{t=t_{0}}+\left.\frac{\Delta \delta^{2}}{2} f_{\delta_{t}}^{\prime \prime}\right|_{t=t_{0}}+\left.\Delta n \Delta \delta f_{n_{t} \delta_{t}}^{\prime \prime}\right|_{t=t_{0}},
$$

or,

$$
f_{n_{t}}^{\prime}+\left(\delta_{t}^{\max }-\delta_{0}\right) f_{\delta_{t}}^{\prime}+\frac{1}{2} f_{n_{t}}^{\prime \prime}+\frac{\left(\delta_{t}^{\max }-\delta_{0}\right)^{2}}{2} f_{\delta_{t}}^{\prime \prime}+\left(\delta_{t}^{\max }-\delta_{0}\right) f_{n_{t} \delta_{t}}^{\prime \prime}=\mathbf{I}_{0}-1,
$$

where all partial derivatives are taken at $t=t_{0}$. It is not difficult to observe that (23) is a quadratic function with only variable $\delta_{t}^{\max }$ to determine. Therefore, we could easily derive its close-form expression as:

$$
\delta_{t}^{\max }=\delta_{0}+\frac{-\left(f_{n_{t} \delta_{t}}^{\prime \prime}+f_{\delta_{t}}^{\prime}\right)+\sqrt{\left(f_{n_{t} \delta_{t}}^{\prime \prime}+f_{\delta_{t}}^{\prime}\right)^{2}-2 f_{\delta_{t}}^{\prime \prime}\left(2 f_{n_{t}}^{\prime}+f_{n_{t}}^{\prime \prime}+2-2 \mathbf{I}_{0}\right)}}{f_{\delta_{t}}^{\prime \prime}},
$$

where partial derivatives $f_{n_{t}}^{\prime}, f_{\delta_{t}}^{\prime}, f_{n_{t} \delta_{t}}^{\prime \prime}, f_{n_{t}}^{\prime \prime}$, and $f_{\delta_{t}}^{\prime \prime}$ are taken at $\left(n_{0}, \delta_{0}\right)$ as inputs when $t=t_{0}$.

\subsection{Admission Control for Missions}

The first step for making an admission decision for this new chemical detection task is to use earlier derived ratio $l$ in (8) to capture the abrupt index change due to the new mission arrival and later, any completion event of existing missions. As mentioned, the impact of admitting one more mission on existing ones' QoI could be captured by the abrupt QoI index change from $\mathbf{I}_{0}$ to $l \cdot \mathbf{I}_{0}$, as shown in Figure 4(a) and 4(c). If the required QoI is satisfied, condition

$$
l \cdot \mathbf{I}_{0} \leq 1
$$

has to hold, otherwise it is not satisfactory.

The second step is to compare the QoI requirement of the newly arrived mission with the sensor network capacity bound. As in this example, we have derived the sensor network capacity in terms worst case confidence bound as in (24), or:

$$
\mathbf{c}_{t}^{\max }=\delta_{t}^{\max } .
$$

Suppose a new chemical detection mission $q$ is assigned with different QoI requirements as error bound $\epsilon_{q}^{r}$ and confidence $\delta_{q}^{r}$. Then, the admission decision variable $\Upsilon_{q}$ is chosen according to the following:

$$
\Upsilon_{q}=\left\{\begin{array}{ll}
\text { Admission, } & \text { if } l \cdot \mathbf{I}_{0} \leq 1 \text { and } \mathbf{c}_{t}^{\max }=\delta_{t}^{\max } \geq \delta_{q}^{r}, \\
\text { Rejection, } & \text { otherwise }
\end{array},\right.
$$

i.e., if the abrupt change of QoI satisfaction index is still below the index bound, and the maximum supportable confidence bound for a new mission is bigger than mission q's QoI requirement, then the mission is admitted; otherwise, it is rejected.

\section{CONCLUSIONS}

In this paper, a new approach to designing the architecture of WSNs for mission-oriented applications was proposed, where detailed interactions among mission's multiple QoI requirements, sensor network capacity, and admission control for missions were modeled and analyzed. First, a novel concept of QoI satisfaction index experienced by each QoI requirement, namely, accuracy, completeness, and timeliness, was introduced. Second, multiple indexes were integrated into one single value that represents the degree of satisfaction for all missions in the network. Third, given different operational contexts of different QoI requirements such as geographical areas, time, and available network resources, we captured the potential abrupt QoI satisfaction index change due to mission arrivals and completions. Fourth, by considering the QoI requirements as inputs and observable QoI satisfaction index as an output, the whole network was modeled as a black box, where Taylor expansion was used as a tool to analyze the sensor network capacity. Last, admission decisions for new missions were made based on this capacity and changed QoI satisfaction index. Finally, a comprehensive chemical detection example was given to illustrate the usefulness of our framework as well as important system parameters. 


\section{ACKNOWLEDGMENTS}

This research was sponsored by US Army Research laboratory and the UK Ministry of Defence and was accomplished under Agreement Number W911NF-06-3-0001. The views and conclusions contained in this document are those of the authors and should not be interpreted as representing the official policies, either expressed or implied, of the US Army Research Laboratory, the U.S. Government, the UK Ministry of Defense, or the UK Government. The US and UK Governments are authorized to reproduce and distribute reprints for Government purposes notwithstanding any copyright notation hereon.

\section{REFERENCES}

[1] Akyildiz, I., Su, W., Sankarasubramaniam, Y., and Cayirci, E., "A survey on sensor networks," IEEE Comm. Mag. 40, 102-114 (Aug 2002).

[2] Wang, R. Y. and Strong, D. M., "Beyond accuracy: What data quality means to data consumers," $J$. Management Information Systems (Spring 1996).

[3] Johnson, M. E. and Chang, K. C., "Quality of information for data fusion in net centric publish and subscribe architectures," in [8th Int'l Conf. on Information Fusion (FUSION 2005)], (July 25 - 29 2005).

[4] Bisdikian, C., Branch, J., Leung, K. K., and Young, R. I., "A letter soup for the quality of information in sensor networks," in [IEEE Information Quality and Quality of Service (IQ2S) Workshop (in IEEE PerCom'09)], (March 2009).

[5] Tolstikov, A., Biswas, J., and Tham, C.-K., "Data loss regulation to ensure information quality in sensor networks," in [Int'l Conf. on Intelligent Sensors, Sensor Networks and Information Processing Conference, 2005.], 133-138 (Dec. 2005).

[6] Tolstikov, A., Tham, C.-K., and Biswas, J., "Quality of information assurance using phenomena-aware resource management in sensor networks," in [14th IEEE Int'l Conf. on Networks, ICON'06.], 1, 1-7 (Sept. 2006).

[7] Henricksen, K. and Robinson, R., "A survey of middleware for sensor networks: state-of-the-art and future directions," in [Int'l Workshop on Middleware for sensor networks, MidSens '06], 60-65 (2006).

[8] Heinzelman, W., Murphy, A., Carvalho, H., and Perillo, M., "Middleware to support sensor network applications," IEEE Network 18, 6-14 (Jan/Feb 2004).

[9] Lazaridis, I., Han, Q., Yu, X., Mehrotra, S., Venkatasubramanian, N., Kalashnikov, D. V., and Yang, W., "Quasar: quality aware sensing architecture," SIGMOD Rec. 33(1), 26-31 (2004).

[10] Alex, H., Kumar, M., and Shirazi, B., "Midfusion: middleware for information fusion in sensor network applications," in [Intelligent Sensors, Sensor Networks and Information Processing Conf., 2004], 617-622 (Dec. 2004).

[11] Heckerman, D., "A tutorial on learning bayesian networks," tech. rep., the ACM Communications (1995).

[12] Zhang, Y. and Ji, Q., "Active and dynamic information fusion for multisensor systems with dynamic bayesian networks," IEEE Trans. on Systems, Man, and Cybernetics, Part B 36, 467-472 (April 2006).

[13] Tolstikov, A., Xiao, W., Biswas, J., Zhang, S., and Tham, C.-K., "Information quality management in sensor networks based on the dynamic bayesian network model," in [3rd Int'l Conference on Intelligent Sensors, Sensor Networks and Information, ISSNIP 200\%.], 751-756 (Dec. 2007).

[14] Liu, C. H., Gkelias, A., and Leung, K. K., "Connection admission control and grade of service for qos routing in mesh networks," in [IEEE PIMRC 2008], (Sept. 2008).

[15] Zhu, H., Li, V. O. K., Ma, Z., and Zhao, M., "Statistical connection admission control framework based on achievable capacity estimation," in [IEEE ICC 2006], 2, 748-753 (June 2006).

[16] Apostol, T., [Calculus], Jon Wiley \& Sons, Inc. (1967). 